\title{
BMJ Open Pharmacological and non- pharmacological treatments for major depressive disorder: review of systematic reviews
}

\author{
Gerald Gartlehner, ${ }^{1,2}$ Gernot Wagner, ${ }^{1}$ Nina Matyas, ${ }^{1}$ Viktoria Titscher, ${ }^{1}$ \\ Judith Greimel, ${ }^{3}$ Linda Lux, ${ }^{2}$ Bradley N Gaynes, ${ }^{4}$ Meera Viswanathan, ${ }^{2}$ \\ Sheila Patel, ${ }^{2}$ Kathleen N Lohr ${ }^{2}$
}

To cite: Gartlehner G, Wagner G, Matyas N, et al. Pharmacological and non-pharmacological treatments for major depressive disorder: review of systematic reviews. BMJ Open 2017;7:e014912. doi:10.1136/ bmjopen-2016-014912

- Prepublication history and additional material for this paper are available online. To view these files please visit the journal online (http://dx.doi. org/10.1136/bmjopen-2016014912).

Received 1 November 2016 Revised 16 March 2017 Accepted 27 April 2017

\section{(a) CrossMark}

${ }^{1}$ Department for Evidencebased Medicine and Clinical Epidemiology, Danube University Krems, Krems, Austria

${ }^{2}$ RTI-University of North Carolina Evidence-based Practice Center, RTI International, North Carolina, USA

${ }^{3}$ University of Hohenheim, Stuttgart, Germany

${ }^{4}$ Department of Psychiatry, University of North Carolina at Chapel Hill, Chapel Hill, USA

\section{Correspondence to} Professor Gerald Gartlehner; gerald.gartlehner@donau-uni. ac.at

\section{ABSTRACT}

Objectives This study aims to summarise the evidence on more than 140 pharmacological and non-pharmacological treatment options for major depressive disorder (MDD) and to evaluate the confidence that patients and clinicians can have in the underlying science about their effects.

Design This is a review of systematic reviews.

Data sources This study used MEDLINE, Embase, Cochrane Library, PsycINF0 and Epistemonikos from 2011 up to February 2017 for systematic reviews of randomised controlled trials in adult patients with acute-phase MDD.

Methods We dually reviewed abstracts and full-text articles, rated the risk of bias of eligible systematic reviews and graded the strength of evidence.

Results Nineteen systematic reviews provided data on 28 comparisons of interest. For general efficacy, only secondgeneration antidepressants were supported with high strength evidence, presenting small beneficial treatment effects (standardised mean difference: $-0.35 ; 95 \% \mathrm{Cl}$ -0.31 to -0.38 ), and a statistically significantly higher rate of discontinuation because of adverse events than patients on placebo (relative risk (RR) 1.88; 95\% Cl 1.0 to 3.28 ). Only cognitive behavioural therapy is supported by reliable evidence (moderate strength of evidence) to produce responses to treatment similar to those of second-generation antidepressants $(45.5 \%$ vs $44.2 \%$; RR $1.10 ; 95 \% \mathrm{Cl} 0.93$ to 1.30). All remaining comparisons of non-pharmacological treatments with second-generation antidepressants either led to inconclusive results or had substantial methodological shortcomings (low or insufficient strength of evidence).

Conclusions In contrast to pharmacological treatments, the majority of non-pharmacological interventions for treating patients with MDD are not evidence based. For patients with strong preferences against pharmacological treatments, clinicians should focus on therapies that have been compared directly with antidepressants.

Trial registration number International Prospective Register of Systematic Reviews (PROSPER0) registration number: 42016035580.

\section{INTRODUCTION}

Major depressive disorder (MDD) ${ }^{1}$ is the most prevalent and disabling form of depression,
Strengths and limitations of this study

- This is the first review of systematic reviews assessing the benefits and harms of more than 140 pharmacological and non-pharmacological treatments for major depressive disorder.

- We used rigorous systematic review and novel graphical methods to summarise treatment effects and present the strength of the underlying evidence.

- Like any review of systematic reviews, we could draw conclusions only about interventions that had been assessed by systematic reviews.

- We did not take combination or augmentation strategies of antidepressants with nonpharmacological interventions into consideration, but in clinical practice, this is a common treatment strategy.

affecting more than 30 million Europeans per year. ${ }^{2}$ In the USA, the estimated lifetime prevalence of MDD is $16 \%{ }^{3}$ In addition to its burden of disease, MDD exerts a negative impact on physical health ${ }^{4-7}$ and adherence to medical treatment. ${ }^{89}$

Second-generation antidepressants (eg, selective serotonin reuptake inhibitors or selective serotonin norepinephrine reuptake inhibitors) are the most commonly used treatments for acute MDD ${ }^{10}$ Most evidence-based guidelines recommend these medications as a first-step therapy. ${ }^{11} 12$

Nevertheless, patients with depression may prefer non-pharmacological options because antidepressant therapies also come with considerable risks for harms. Up to $63 \%$ of patients on second-generation antidepressants experience adverse events; between $7 \%$ and $15 \%$ of patients discontinue treatment because of adverse events. ${ }^{13}$ Concerns about the 'addictiveness' of antidepressants are also a common reason for patients' scepticism 
about prescription medications ${ }^{14}{ }^{15}$; women and ethnic minorities, in particular, often prefer non-pharmacological options as first-step treatments of depression. ${ }^{16} 17$ Antidepressants also have a substantially higher treatment-specific stigma than, for example, herbal remedies. ${ }^{18}$

Such scepticism toward antidepressants reflects a general trend toward 'natural treatments' throughout medicine. In 2012, an estimated 59 million persons in the USA spent US $\$ 30.2$ billion in out-of-pocket expenses on some type of complementary health approach. ${ }^{19}$ In a survey of psychiatric patients, more than half of patients with self-reported depressive disorders used complementary and alternative medicine (CAM) therapies. ${ }^{20}$

Non-pharmacological treatment options for depression are vast. The Cochrane Depression and Neurosis Group lists 87 psychological interventions ${ }^{21}$; a comprehensive summary from an Australian patient advocacy group catalogued 56 CAM interventions for the treatment of depression (beyondblue: A guide to what works for depression (http://resources.beyondblue.org.au/prism/file? token=BL/0556)) .

Because of the multitude of non-pharmacological options, for clinicians, the great challenge is how to balance patients' interest in alternatives to medications with the professional responsibility to choose treatments that are supported by scientific evidence.

The goal of this project was to provide an overview of the general efficacy and risk of harms of pharmacological and non-pharmacological interventions for treating patients with MDD. Furthermore, we strove to compare benefits and harms of non-pharmacological interventions with second-generation antidepressants as the most common treatments for acute-phase MDD.

\section{METHODS}

A review of systematic reviews is designed to compile evidence from multiple systematic reviews of interventions into one accessible, usable document. ${ }^{22}$ We registered the protocol in PROSPERO (International Prospective Register of Systematic Reviews; registration number: 42016035580).

\section{Populations, interventions, comparators, outcomes, timing and settings}

table 1 presents eligibility criteria for populations, interventions, comparators, outcomes, timing and settings of systematic reviews and meta-analyses. In this table, the term 'articles' refers to any systematic reviews or meta-analyses of randomised controlled trials (RCTs) published in peer-reviewed journals or other sources. We limited the publication period to 2011 or later because methods research indicates that more than $50 \%$ of systematic reviews are outdated 5.5 years after publication. ${ }^{23}$

For eligible psychological interventions, we used the Cochrane Depression and Neurosis Group classification. ${ }^{21}$ For CAM, we were interested in any intervention that the non-profit patient advocacy group beyondblue listed as a 'non-medical' intervention for treating depressed patients. ${ }^{24}$ Online supplementary file 1 lists the 87 eligible psychological interventions and the 56 eligible CAM interventions.

\section{Literature searches}

To identify relevant systematic reviews or meta-analyses, we searched MEDLINE (via PubMed), Embase, the Cochrane Library, PsycINFO and Epistemonikos. We used both index terms (eg, Medical Subject Headings, Emtree) and free-text keywords to search for MDD. We limited the electronic searches to 'human,' 'English, German or Italian language,' 'adults' and systematic reviews or meta-analyses. We searched sources from 1 January 2011 to 20 February 2017.

We imported all citations into an electronic database (EndNote X.6.0.1). The search strategies and yields of the searches are given in online supplementary file 2 .

\section{Screening process}

We developed and pilot-tested review forms using the eligibility criteria in table 1 . In a two-stage review process, two persons independently reviewed abstracts and fulltext articles. We resolved discrepancies by consensus or by consulting a third senior investigator. For each comparison and outcome, we chose a single systematic review providing the best available evidence. If more than one systematic review on the same intervention met eligibility criteria, we chose the review with (1) the lowest risk of bias, (2) the most recent search date and (3) the most comprehensive scope. For each eligible systematic review, we determined whether RCTs included in it also met our inclusion criteria (see table 1).

\section{Data abstraction}

We designed and used a structured form to ensure consistency of data abstraction. If all studies in a systematic review met our eligibility criteria, we extracted summary estimates from meta-analyses. If one or more studies did not meet our eligibility criteria, we extracted data from individual studies. For example, when systematic reviews included mixed populations with different depressive disorders, we retrieved individual publications on patients with MDD. When data were unclear or contradictory, we contacted review authors for clarification. A second senior reviewer evaluated the completeness and accuracy of the data abstraction.

\section{Risk of bias assessment}

To assess methodological limitations (risk of bias) of eligible systematic reviews, we used the AMSTAR (Assessing Methodological quality of Systematic Reviews) tool. ${ }^{25}$ Two independent reviewers assigned ratings for study limitations. They resolved any disagreements by consensus or by consulting a third independent party. For the risk of bias of individual studies in a systematic review, we relied on the ratings of the original reviews' authors. AMSTAR ratings of included studies are given in online supplementary file 3 . 
Table 1 Study eligibility criteria: populations, interventions, comparators, outcomes, timing and settings for the review of reviews (PICOTS)

\begin{tabular}{|c|c|}
\hline PICOTS & Specific inclusion or exclusion criteria \\
\hline Population & $\begin{array}{l}\text { Adult (18 years and above) patients of all races and ethnicities with MDD who are undergoing first-step } \\
\text { treatment during acute treatment phase. } \\
\text { We did not include populations with bipolar disorder, perinatal depression, dysthymia, seasonal affective } \\
\text { disorder or subsyndromal depression. We also did not include populations exclusively comprising patients } \\
\text { with medical comorbidities and depression (eg, populations with heart disease and depression or with } \\
\text { cancer and depression) }\end{array}$ \\
\hline Outcomes & $\begin{array}{l}\text { Efficacy and effectiveness: response, change of depression scores } \\
\text { Adverse events (safety and tolerability): overall discontinuation, discontinuation because of adverse events }\end{array}$ \\
\hline Timing & No restrictions \\
\hline Setting & All settings \\
\hline Time period & Articles published in 2011 and later \\
\hline Study design & $\begin{array}{l}\text { Systematic reviews }{ }^{\star} \text { and meta-analyses (if based on a systematic review) of RCTs published in English, } \\
\text { German or Italian languages }\end{array}$ \\
\hline
\end{tabular}

*Systematic reviews are defined based on the Cochrane handbook as a literature review that attempts to collate all empirical evidence using (a) clearly stated objectives and predefined eligibility criteria, (b) an explicit reproducible methodology, (c) a systematic search, (d) an assessment of the validity of the findings of the included studies and (e) a systematic presentation, and synthesis, of the characteristics and findings of the included studies. ${ }^{22}$

CAM, complementary and alternative medicine; MDD, major depressive disorder; RCT, randomised controlled trial.

\section{Evidence synthesis}

Our aim was to depict the magnitude of beneficial and harmful treatment effects and the confidence that patients and clinicians can have in the underlying science about these effects. We used effect estimates of systematic reviews if all included RCTs met our eligibility criteria. In instances where individual RCTs of eligible systematic reviews did not meet our eligibility criteria (eg, because they used treatment as usual as a control group), we recalculated quantitative analyses removing ineligible studies.

For general efficacy, we were interested in the improvement of depressive symptoms. We present standardised mean differences because methods of assessments differed substantially across systematic reviews. A 
standardised mean difference of 0 indicates that both groups had similar improvements; effects of -0.5 or -1 indicate that $69 \%$ or $84 \%$ of patients in the intervention group, respectively, had greater reductions on depression scores than the average patient in the control group. For the risk of harms, we present overall discontinuation rates and discontinuation rates because of adverse events.

For the comparative efficacy of non-pharmacological treatments with second-generation antidepressants, we used relative risk (RR) of response to treatment (as defined by the authors but most commonly presented as a $50 \%$ reduction of symptoms on a depression rating scale). If necessary, we recalculated RR so that a value below 1 would represent fewer responses of patients using non-pharmacological treatments and a value greater than 1 would represent more responses. We present treatment effects also as absolute risk reductions or increases (differences in numbers of patients who respond to treatment, per 1000 treated patients) with the related $95 \%$ CIs.

\section{Quantitative analyses}

As described above, in instances where individual RCTs of eligible systematic reviews did not meet our eligibility criteria, we recalculated quantitative analyses removing ineligible studies. To summarise data quantitatively, we followed established guidance. ${ }^{26}$ For all analyses, we used both random-effects and fixed-effects models. We report results of random-effects analyses (DerSimonian \& Laird). In general, the findings from the random-effects and fixed-effects analyses were similar. We assessed statistical heterogeneity between studies by calculating the $\chi^{2}$ statistic and Cochran's q. We used the $\mathrm{I}^{2}$ statistic (the proportion of variation in study estimates attributable to heterogeneity) to estimate the magnitude of heterogeneity. We examined potential sources of heterogeneity using sensitivity analyses and assessed publication bias with funnel plots and Kendall's tests.

For general efficacy, we estimated standardised mean differences using Hedges' g. ${ }^{27}$ If systematic reviews presented effect sizes as Cohen's d, we used a correction factor $(J)$ to convert to Hedges' $g$ : $\left(J=1-\frac{3}{4 d f-1}\right)$, where df stands for 'degrees of freedom'.

If systematic reviews presented effect estimates of general efficacy as dichotomous outcomes, we calculated $\log$ ORs and converted them first to Cohen's d $\left(\mathrm{d}=\log \mathrm{OR} \frac{\sqrt{3}}{\pi}\right)$ and then to Hedges' $\mathrm{g}$ using the correction factor presented above. For each estimate, we calculated variances and CIs.

For all statistical calculations, we used Microsoft Excel (V.2010, Microsoft, Redmond, WA, USA) or Review Manager 5.3 (V.5.3. Copenhagen, The Cochrane Collaboration, 2014).

\section{Strength of the evidence}

We graded the strength of evidence based on guidance for AHRQ Evidence-based Practice Centres on the use of GRADE (Grading of Recommendations Assessment, Development and Evaluation) Working Group. ${ }^{28} 29$
Strength of evidence can take four grades: high, moderate, low or insufficient. We considered grades of high or moderate strength as reliable evidence.

\section{RESULTS}

Searches detected 2532 citations; 19 systematic reviews met our eligibility criteria and provided the most recent summaries of evidence on 28 comparisons of interest. ${ }^{30-44}$ Thirty-one additional systematic reviews formally met eligibility criteria, but their content was superseded by at least one of the 19 reviews mentioned above (online supplementary file 4). figure 1 presents the flow of the literature; table 2 presents characteristics of included reviews.

For the majority of non-pharmacological treatments, we did not find any systematically appraised evidence.

In the following sections, we first provide an overview of treatment effects of non-pharmacological and common pharmacological treatments compared with inactive interventions.

We then present results on the comparative benefits and harms of non-pharmacological interventions and second-generation antidepressants.

\section{Non-pharmacological and pharmacological treatments compared with inactive interventions}

\section{Benefits of treatments}

Sixteen systematic reviews provided data on 17 comparisons with inactive interventions (placebo, sham interventions or waiting list). ${ }^{30-32} 35-37$ 39-43 45-50 figure 2 provides an overview of treatment effects of non-pharmacological and common pharmacological treatments for MDD when compared with inactive interventions using standardised mean differences. The four commonly used pharmacological interventions in the figure are agomelatine, alprazolam, second-generation antidepressants and tricyclic antidepressants.

The comparisons in the figure are ordered by the strength of evidence grades and then alphabetically by the name of the intervention. figure 2 also presents the numbers of trials and the total number of subjects in those trials; thus, the size of the circles reflects the numbers of participants (on a logarithmic scale). Online supplementary file 5 provides detailed strength of evidence ratings.

The only treatments for acute-phase MDD with high strength of evidence were second-generation antidepressants (figure 2). Within this class, the medications rendered modest treatment effects $(-0.35 ; 95 \%$ CI -0.31 to -0.38). Although the dataset included 24 unpublished studies ${ }^{44}$ treatment effects might still be inflated because several methods studies indicate that publication bias is a serious problem in this drug class. ${ }^{51}{ }^{52}$

Reviews on some psychological interventions (third wave cognitive behavioural therapy (CBT) and psychodynamic therapies) reported large treatment effects (third wave CBT: -0.97 ; $95 \%$ CI -0.6 to -1.34 ; psychodynamic therapies: -2.02 ; $95 \%$ CI -0.9 to -3.14 ; low or insufficient strength of evidence, respectively; figure 2). Studies of 


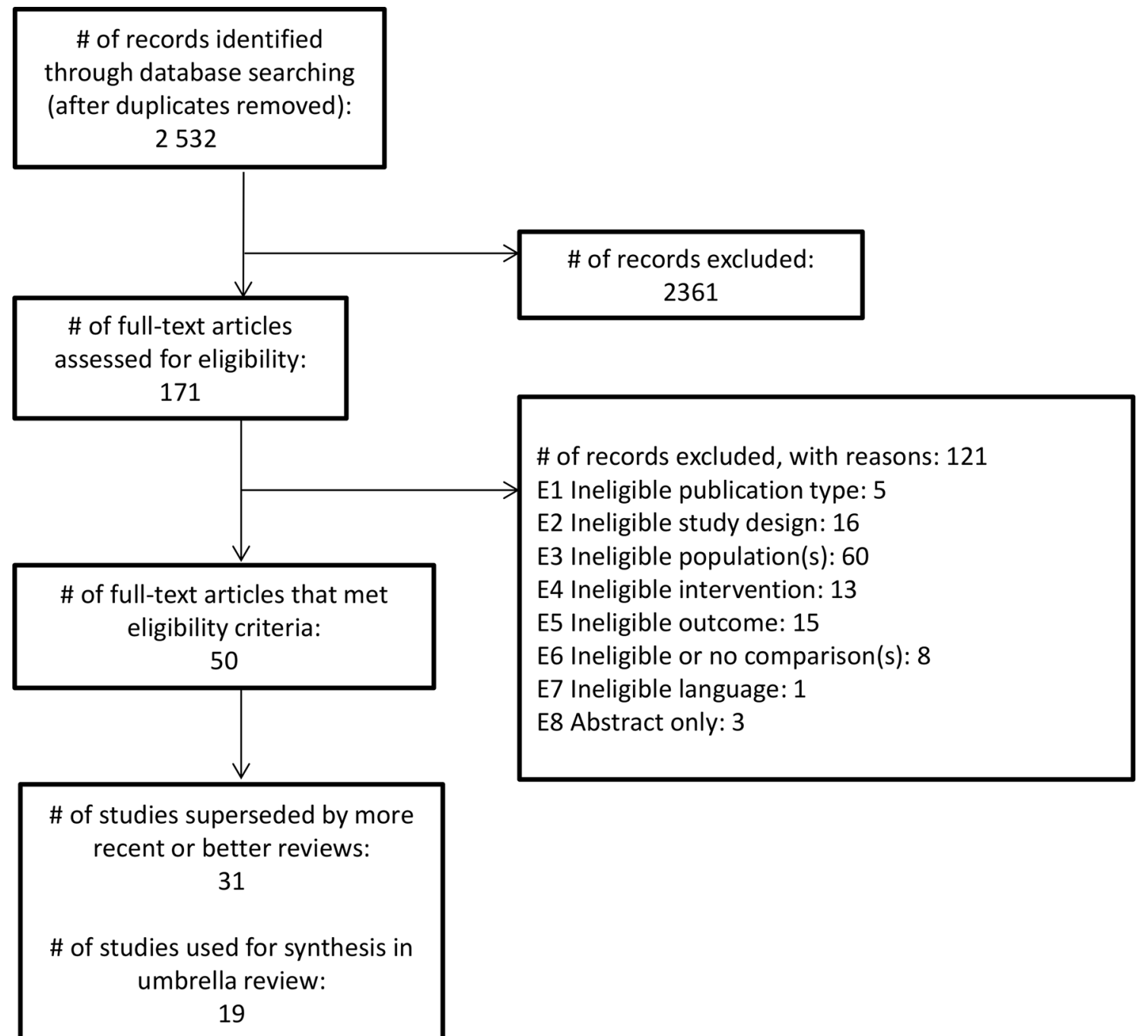

Figure 1 Flow diagram of review of systematic reviews of treatments for major depressive disorder in adults.

these two psychological interventions used waiting lists as control interventions. Patients on waiting lists usually do not experience beneficial placebo effects, which can lead to artificially large treatment effects when active interventions are compared with waiting list controls. Placebo effects in psychiatric populations can be substantial; for example, on average, $35 \%-40 \%$ of patients in doubleblinded trials of antidepressants achieved a response (usually defined as a $50 \%$ reduction of symptoms) to placebo treatment. ${ }^{53}$

For many of the therapies in figure 2, the types of inactive comparators varied and involved different magnitudes of placebo effects. Consequently, comparisons of treatment effects across different interventions have to be made cautiously.

Risk of harms

Information on overall discontinuation and discontinuation because of adverse events was scarce. figure 3 depicts the absolute risk reductions or increases for overall discontinuation and discontinuation because of adverse events-namely, the bars showing the 95\% CIs of either fewer or more discontinuations per 1000 patients.
Only patients on second-generation antidepressants had a statistically significantly higher rate of discontinuation because of adverse events than patients on placebo $(4.5 \%$ vs $2.6 \%$; RR $1.88,95 \%$ CI 1.07 to 3.28$)$. Most comparisons were of low or insufficient strength of evidence, indicating little certainty in the available effect estimates (details in online supplementary file 5).

\section{Non-pharmacological treatments compared with second-generation antidepressants}

Three systematic reviews provided data on response to treatment for 11 non-pharmacological interventions (four psychological, six CAM and exercise) compared with second-generation antidepressants for the treatment of acute-phase MDD..$^{30}{ }^{31}$ We used response to treatment as defined by authors of the reviews; in most cases, this was a $50 \%$ reduction of symptoms as measured on a depression rating scale (eg, Hamilton Depression Rating Scale). figure 4 depicts the absolute risk reductions or increases for response to treatment per 1000 patients. As in the other figures, the comparisons are ordered by the strength of evidence grades and then alphabetically by the name of the intervention. These estimates are based 


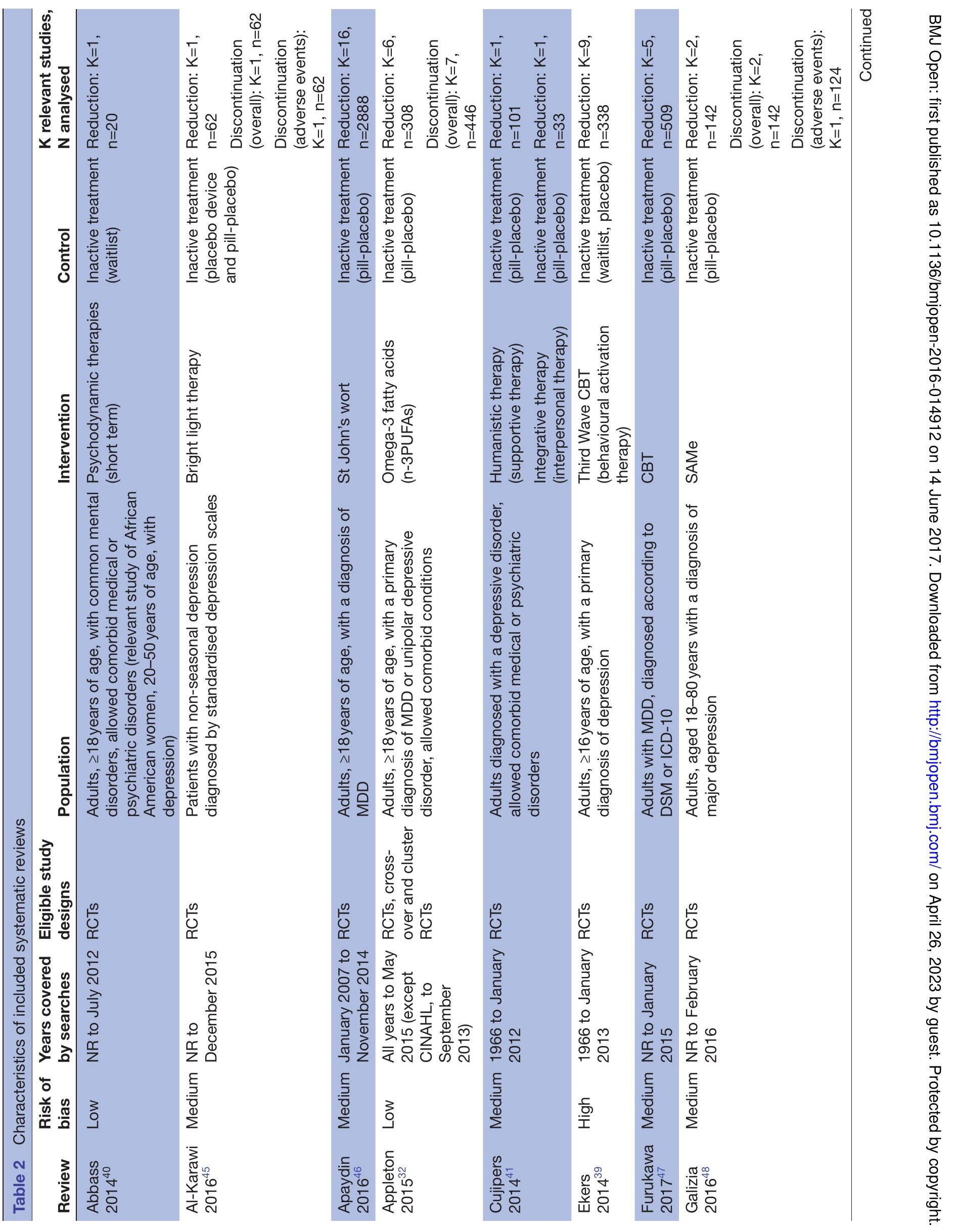




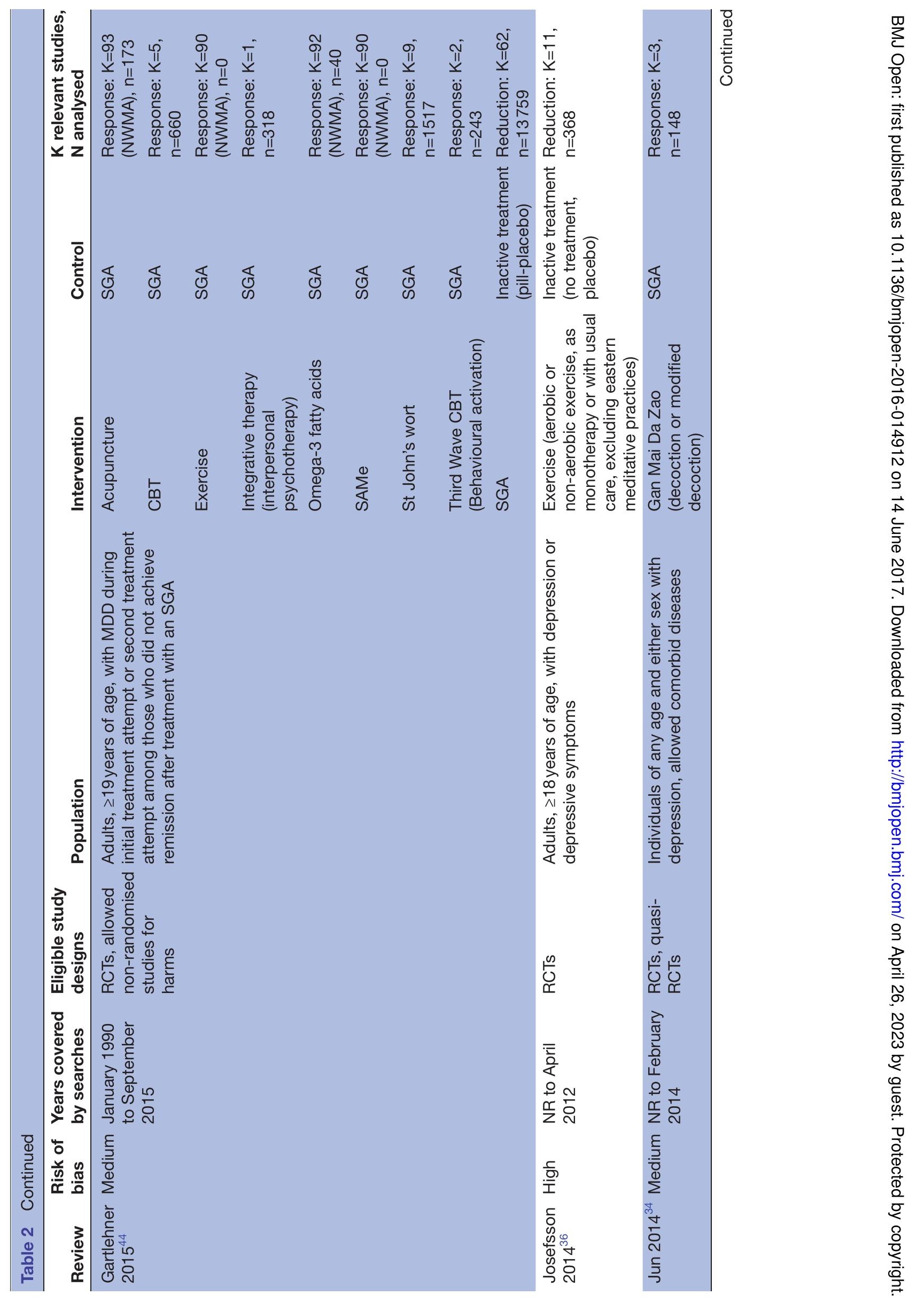




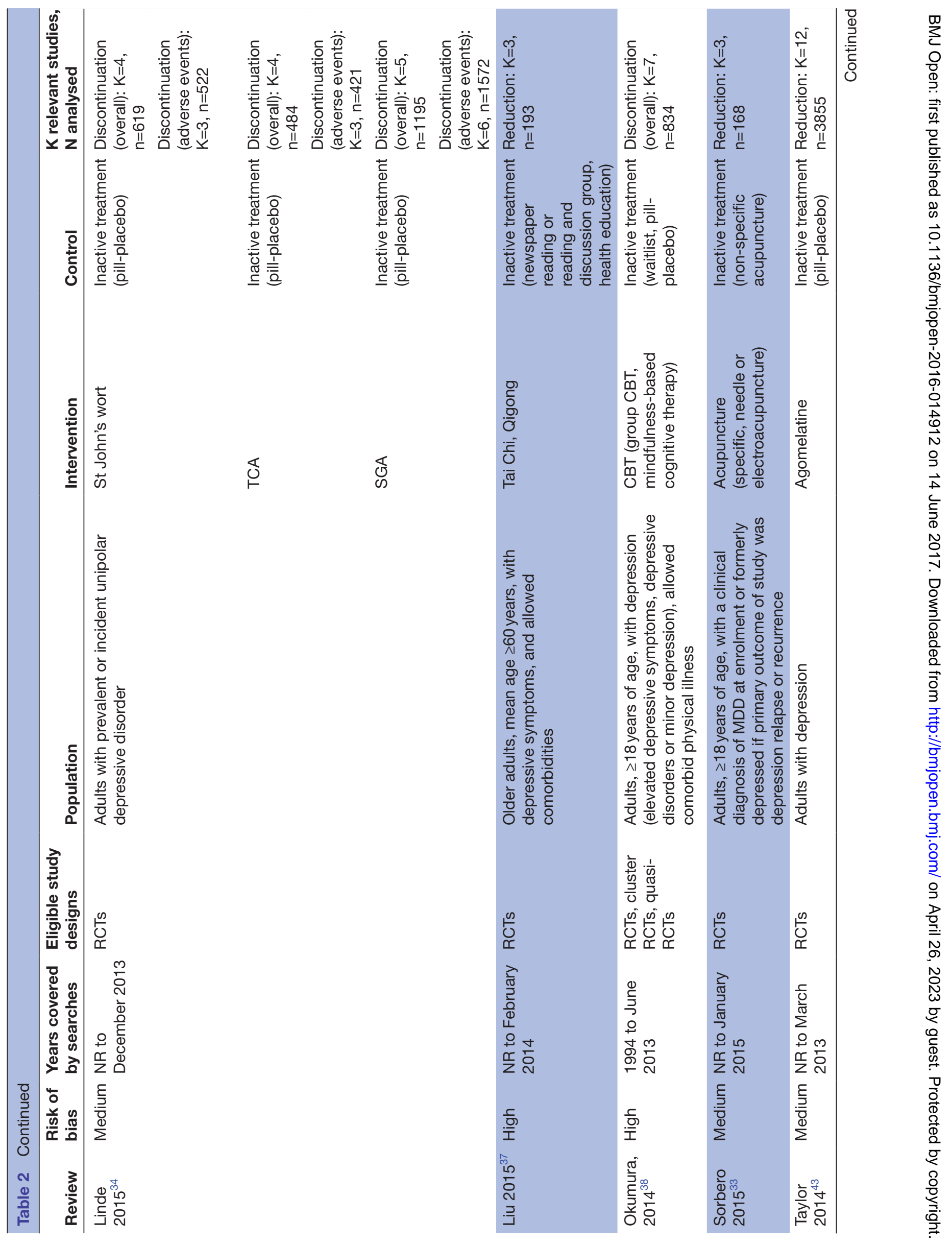




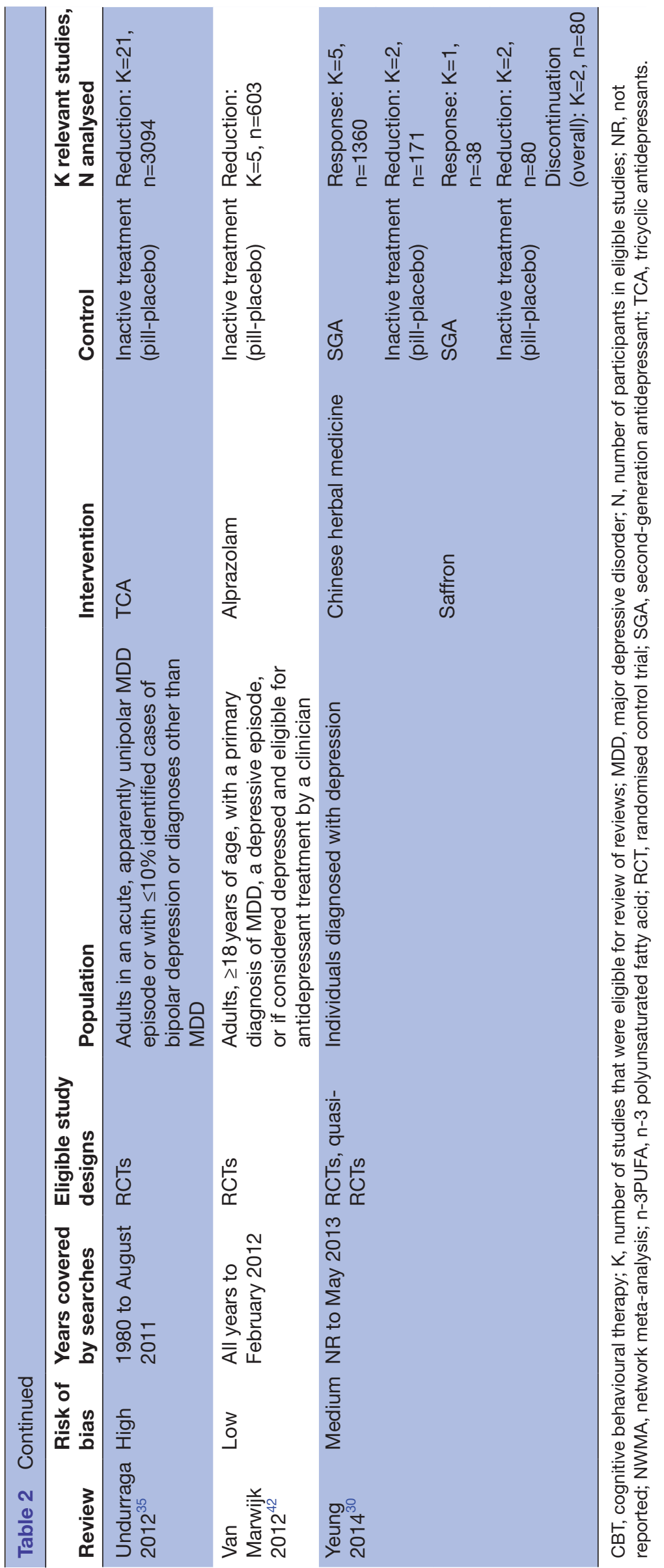




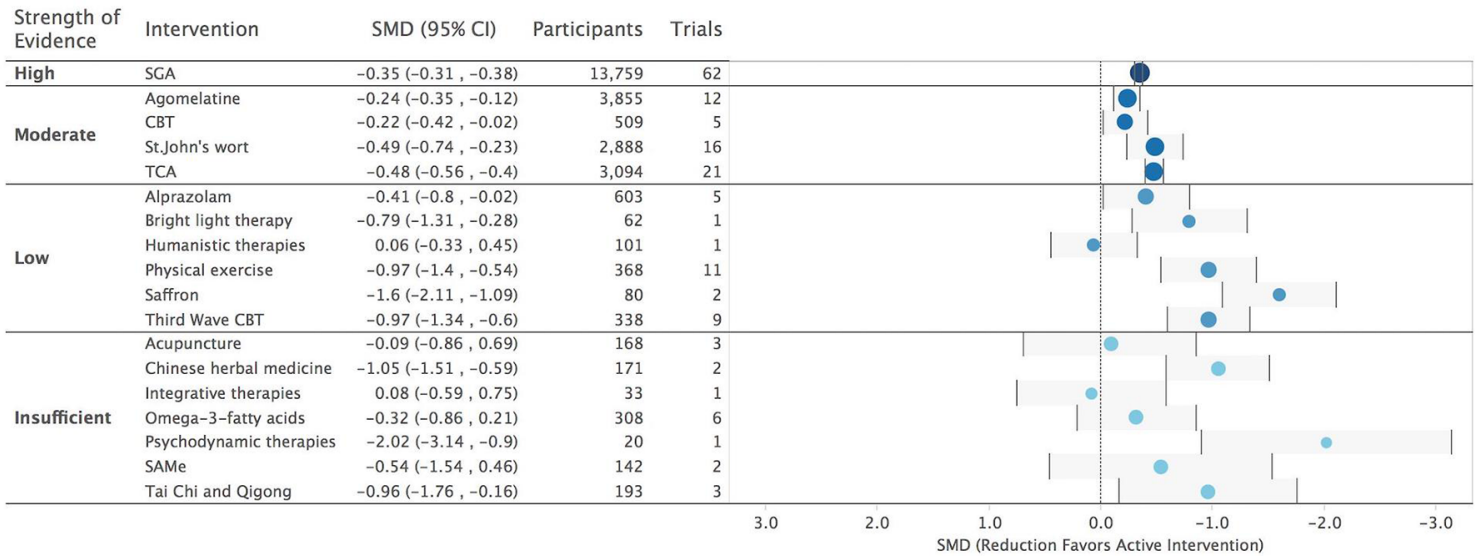

Figure 2 Overview of the strength of evidence of non-pharmacological and pharmacological interventions compared with inactive interventions for the treatment of adult major depressive disorder. CBT, cognitive behavioural therapy; SAMe, S-adenosyl-L-methionine; SGA, second-generation antidepressants; SMD, standardised mean difference; TCA, tricyclic antidepressants.

on meta-analyses or, if meta-analyses were not feasible, on results from the largest and most reliable trial. Online supplementary file 5 provides detailed information on our ratings of strength of evidence domains.

\section{Psychological interventions}

One systematic review reported on the efficacy of four psychological treatments relative to second-generation antidepressants (figure 4); these included CBT, integrative therapies, psychodynamic therapies and third wave $\mathrm{CBT}^{44}$ The most reliable evidence (moderate strength of evidence) compared CBT with second-generation antidepressants. A meta-analysis of five RCTs of low or medium risk of bias with 660 patients provided consistent evidence that the two options had similar efficacy $(45.5 \%$ vs $44.2 \%$; RR 1.10; $95 \%$ CI 0.93 to 1.30$).{ }^{54}$ Including three high risk of bias studies yielded similar results (RR $0.98 ; 95 \%$ CI 0.80 to 1.20$).^{54}$

Integrative therapies also had response rates similar to those for antidepressants (low strength of evidence). ${ }^{44}$ Patients treated with third wave CBT had significantly higher response rates than those on antidepressants, but the strength of evidence was insufficient because of the small sample size and under-dosing of antidepressants in the available trial. No evidence on response was available for psychodynamic therapies, but the available evidence indicated remission rates similar to those for second-generation antidepressants. ${ }^{44}$

\section{CAM interventions}

Three systematic reviews reported on comparisons with second-generation antidepressants for seven (of 56

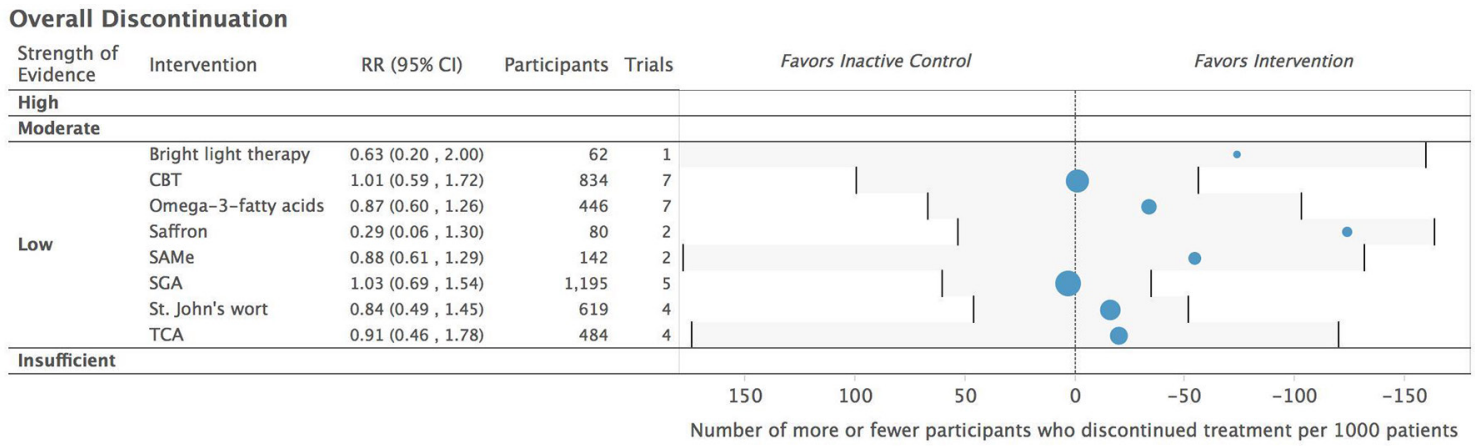

Discontinuation because of Adverse Events

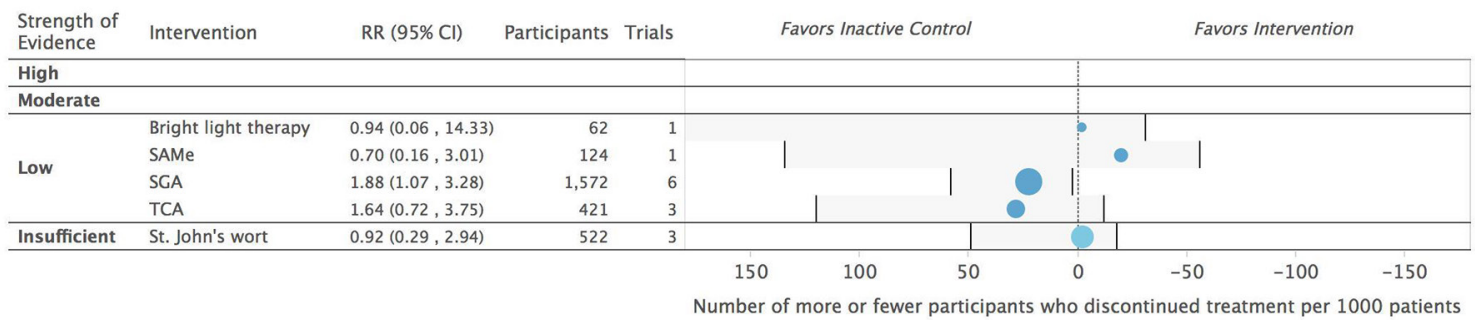

Figure 3 Absolute risk reductions or increases of overall discontinuation or discontinuation because of adverse events comparing non-pharmacological interventions with inactive interventions. CBT, cognitive behavioural therapy; SAMe, S-adenosyl-L-methionine; SGA, second-generation antidepressants; TCA, tricyclic antidepressants. 


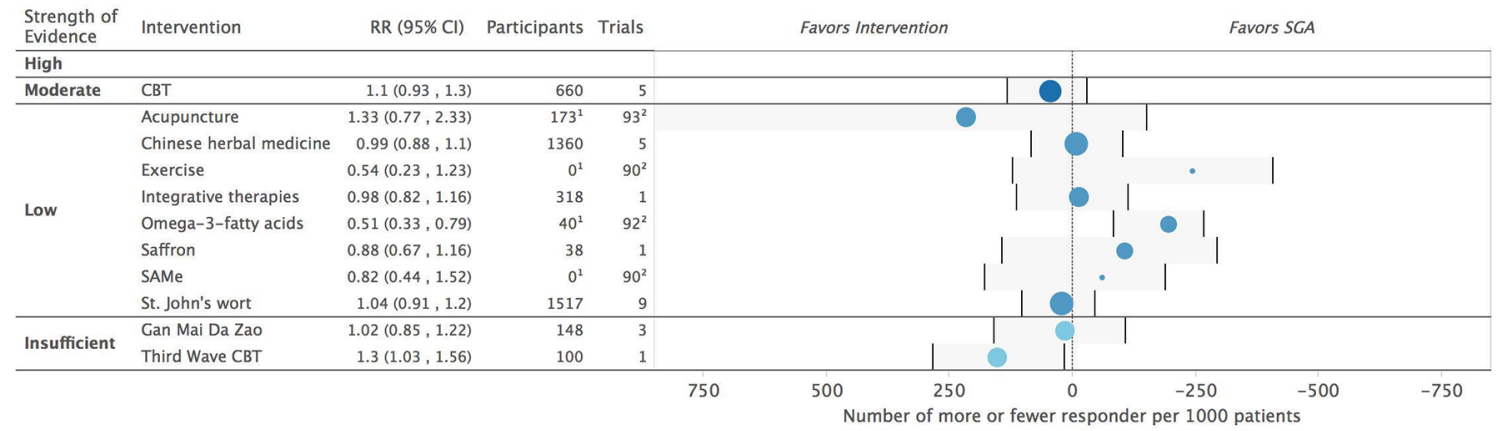

Figure 4 Absolute risk reductions or increases of response to treatment comparing non-pharmacological interventions with second-generation antidepressants for the treatment of adult major depressive disorder. ${ }^{1}$ Number of participants in trials that directly compared intervention with second-generation antidepressants. ${ }^{2}$ Number of trials in network meta-analysis that contributed to the effect estimate. CBT, cognitive behavioural therapy; RR, relative risk; SAMe, S-adenosyl-L-methionine; SGA, second-generation antidepressants.

eligible) CAM interventions-namely, acupuncture, Chinese herbal medicine (without Gan Mai Da Zao), Gan Mai Da Zao, omega-3 fatty acids, S-adenosyl-L-methionine (SAMe), St John's wort and saffron (figure 4). ${ }^{303144}$ Except for omega-3 fatty acids, none of the comparisons yielded statistically significant differences. Based on results of a network meta-analysis, patients using omega-3 fatty acids were statistically significantly less likely to achieve response than patients on antidepressants (RR 0.51; $95 \%$ CI 0.33 to 0.79$).{ }^{44}$ The reliability of results involving CAM interventions, however, is low. Therefore, the lack of statistical significance of most comparisons should not be interpreted as equivalence of treatment effects.

Some comparisons had wide CIs (eg, acupuncture, Gan Mai Da Zao, SAMe, saffron) rendering inconclusive findings about the comparative efficacy of treatments. Other comparisons had more precise results (eg, Chinese herbal medicine or St John's wort) but severe methodological shortcomings. For example, several trials of $\mathrm{St}$ John's wort used moderate-dose or low-dose second-generation antidepressant regimens as comparators, not fully using the approved range of antidepressant doses. ${ }^{44}$ Two of five trials comparing Chinese herbal medicine with antidepressants had serious design or analytic limitations such as flawed randomisation or lack of allocation concealment. $^{30}$

\section{Exercise}

A network meta-analysis produced inconclusive results about differences in response rates between physical exercise and second-generation antidepressants (figure 4). ${ }^{44}$

\section{Comparative harms}

The discontinuation of treatment because of adverse events were generally lower for patients treated with non-pharmacological interventions than for those receiving second-generation antidepressants, although differences did not always reach statistical significance. Patients on St John's wort had a statistically significantly lower rate of discontinuation because of adverse events (3.8\% vs $6.8 \%$; RR 0.59 ; $95 \%$ CI 0.38 to 0.89$).{ }^{44}$ Patients on any psychological treatment had a numerically lower risk for discontinuation of treatment because of adverse events (2.1\% vs 7.1\%; RR 0.37; $95 \%$ CI 0.12 to 1.12$).{ }^{44}$ Likewise, patients who used physical exercise discontinued treatment because of adverse events less often than those treated with antidepressants $(0 \%$ vs $6 \%$; RR 0.15 ; $95 \%$ CI 0.01 to 2.86 ), but the difference did not reach statistical significance. ${ }^{44}$ Little evidence on treatment discontinuation was available for most CAM interventions, particularly for Chinese herbal medicine or saffron. ${ }^{30} 31$

\section{DISCUSSION}

Out of more than 140 interventions of interest, our review identified only five treatments for which the general efficacy for acute-phase MDD is supported by reliable evidence (ie, evidence graded as high or moderate strength of evidence). Among those, CBT is the only psychological intervention and St John's wort is the only CAM intervention. For the vast majority of non-pharmacological interventions, either no systematic review evidence was available or the certainty of the evidence was severely limited. When compared with second-generation antidepressants, only CBT had similar efficacy based on moderate strength evidence. Overall, our analyses highlighted a lack of robust evidence for the majority of non-pharmacological treatments.

To our knowledge, our study was the first review of systematic reviews assessing more than 140 interventions for treating adults with MDD. It provides a unique synthesis of the available, systematically appraised evidence on these treatment options, beyond the individual reviews on depression therapies that have been published over the past decade.

Our study does have several limitations, however. First and most importantly, like any review of systematic reviews, we could draw conclusions only about interventions that had been assessed by systematic reviews. Conceivably, RCTs are available for some interventions that have never been evaluated systematically in a review. Therefore, the absence of systematic reviews cannot be equated with an absence of RCTs. In addition, eligibility criteria of these reviews sometimes included only a subset of available studies (eg, studies conducted in primary care settings). Such reviews do not provide a picture of the totality of the evidence but sometimes 
were the only ones that were available on a specific comparison of interest. Second, reviews of systematic reviews rely on results from other investigators. Although most of the reviews had few problems in methods, conceivably these authors did miss some RCTs. Likewise, we relied on the risk of bias appraisals of RCTs that authors of included systematic reviews had done. Most reviews used two independent reviewers to rate risk of bias; double checking their ratings was beyond the scope of our study. Third, reporting of characteristics of populations, interventions, comparators and outcomes in included systematic reviews was often suboptimal. Frequently, we could not tell with certainty whether included populations were exclusively adult patients with acute-phase MDD; sometimes, we could not determine the exact control interventions that authors had combined in their meta-analyses. We did not take several meta-analyses into consideration that combined studies with inactive treatments and treatment as usual as control interventions. Because treatment as usual cannot be viewed as 'inactive,' we believe that such meta-analyses will lead to biased results. Fourth, as in any literature review, the reliability of our results is directly related to number of available studies and their quality. Some of the systematic reviews included only few studies with few events. The strength of evidence grades reflect these concerns and the certainty of our results; for most cases, these grades were low or insufficient. Such low strength of evidence indicates that future studies might have a substantial impact on the effect estimates reported in our review. Furthermore, we had no way to assess how meta-biases such as reporting biases or funding biases could have affected our findings. Finally, we did not take combination or augmentation strategies of antidepressants with non-pharmacological interventions into consideration, but in clinical practice, this is a common treatment strategy.

We believe that our results may have important clinical implications. They provide patients and clinicians with solid and up-to-date information about which treatment options have (or have not) been evaluated in rigorous systematic reviews. For patients with strong preferences against pharmacological treatment, clinicians can offer therapies that have been compared directly with antidepressants. CBT, for example, is a well-supported, first-step alternative to pharmacological treatment of MDD. Other psychological or CAM interventions might be equally effective, or nearly so, but the evidence base is less reliable. The majority of psychological and CAM interventions, however, are not evidence based; given better alternatives, clinicians should probably advise against them. Such shared and informed decision making might enhance treatment adherence ${ }^{55}$ and could ultimately improve treatment outcomes for patients with MDD. This is especially important because treatment continuity is one of the main challenges in treating such patients. ${ }^{56}$

Our findings also highlight key areas of future research needs. Subsequent trials need to address gaps in our current knowledge about the efficacy of non-pharmacological interventions and about the comparative benefits and harms of pharmacological and non-pharmacological treatments for MDD. In particular, major research gaps pertain to information about the comparative risk of harms and patient-relevant outcomes such as functional capacity and quality of life. For patients and clinicians alike, balancing benefits and harms based on objective information is crucial. Lack of information about harms can lead to a biassed knowledge base and the potential for decisions that cause more harm than good. Future studies should assess benefits and harms with standardised measures to allow for more direct comparisons across studies.

In the end, even in the absence of clearly informative evidence, clinicians and patients need to make decisions. They can discuss what is known and what is not known about the available options to treat MDD, and our work provides a way to start those conversations. For patients with strong preferences against pharmacological treatments, clinicians should focus on therapies that have been compared directly with antidepressants. This review provides a framework to guide discussion of the potential options.

Acknowledgements We would like to thank Monika Kyselova from Danube University Krems and Loraine Monroe from RTI International for administrative support. We are also grateful to Irma Klerings from Danube University Krems for the literature searches and Joshua Green from RTI International for help with data abstraction.

Contributors GG, KL and MV developed the concept of the study; GG, JG, GW, NM and VT conducted the literature review; GW, NM and VT abstracted data and conducted statistical analyses; MV and LL rated the risk of bias of included systematic reviews; GG, GW and NM graded the strength of evidence; BG provided clinical expertise throughout the study; GG and KL wrote the first draft of the manuscript; all authors reviewed the manuscript and provided comments and revisions.

Funding The paper was supported by internal funds from RTI International, Research Triangle Park, North Carolina.

Competing interests None declared.

Provenance and peer review Not commissioned; externally peer reviewed.

Data sharing statement The datasets used for meta-analyses are available from the corresponding author on reasonable request.

Open Access This is an Open Access article distributed in accordance with the Creative Commons Attribution Non Commercial (CC BY-NC 4.0) license, which permits others to distribute, remix, adapt, build upon this work non-commercially, and license their derivative works on different terms, provided the original work is properly cited and the use is non-commercial. See: http://creativecommons.org/ licenses/by-nc/4.0/

(c) Article author(s) (or their employer(s) unless otherwise stated in the text of the article) 2017. All rights reserved. No commercial use is permitted unless otherwise expressly granted.

\section{REFERENCES}

1. American Psychiatric Association. Diagnostic and Statistical Manual of Mental Disorders. 5th ed. VA: Arlington, American Psychiatric Publishing, 2013.

2. Wittchen HU, Jacobi F, Rehm J, et al. The size and burden of mental disorders and other disorders of the brain in Europe 2010. Eur Neuropsychopharmacol 2011;21:655-79.

3. Kessler RC, Berglund P, Demler O, et al. The epidemiology of major depressive disorder: results from the National Comorbidity Survey replication (NCS-R). JAMA 2003;289:3095-105.

4. Fendrich M, Avci O, Johnson TP, et al. Depression, substance use and HIV risk in a probability sample of men who have sex with men. Addict Behav 2013;38:1715-8.

5. Silberbogen AK, Busby AK, Ulloa EW. Impact of psychological distress on prostate cancer screening in U.S. Military Veterans. Am J Mens Health 2014;8:399-408. 
6. McLaughlin KA. The public health impact of Major depression: a call for interdisciplinary prevention efforts. Prev Sci 2011;12:361-71.

7. Farmer A, Korszun A, Owen MJ, et al. Medical disorders in people with recurrent depression. Br J Psychiatry 2008;192:351-5.

8. DiMatteo MR, Lepper HS, Croghan TW. Depression is a risk factor for noncompliance with medical treatment: meta-analysis of the effects of anxiety and depression on patient adherence. Arch Intern Med 2000;160:2101-7.

9. Kessler RC, Bromet EJ. The epidemiology of depression across cultures. Annu Rev Public Health 2013;34:119-38.

10. Mojtabai R, Olfson M. National patterns in antidepressant treatment by psychiatrists and general medical providers: results from the national comorbidity survey replication. J Clin Psychiatry 2008;69:1064-74.

11. Qaseem A, Barry MJ, Kansagara D. Clinical Guidelines Committee of the American College of Physicians. nonpharmacologic versus pharmacologic treatment of adult patients with major depressive disorder: a clinical practice guideline from the American College of Physicians. Ann Intern Med 2016;164:350-9.

12. Jobst A, Brakemeier EL, Buchheim A, et al. European Psychiatric Association guidance on psychotherapy in chronic depression across Europe. Eur Psychiatry 2016;33:18-36.

13. Gartlehner G, Thieda P, Hansen RA, et al. Comparative risk for harms of second-generation antidepressants : a systematic review and meta-analysis. Drug Saf 2008;31:851-65.

14. Churchill R, Khaira M, Gretton V, et al. Treating depression in general practice: factors affecting patients' treatment preferences. Br J Gen Pract 2000;50:905-6.

15. van Schaik DJ, Klijn AF, van Hout HP, et al. Patients' preferences in the treatment of depressive disorder in primary care. Gen Hosp Psychiatry 2004;26:184-9.

16. Cooper LA, Gonzales JJ, Gallo JJ, et al. The acceptability of treatment for depression among African-American, hispanic, and white primary care patients. Med Care 2003;41:479-89.

17. Givens JL, Houston TK, Van Voorhees BW, et al. Ethnicity and preferences for depression treatment. Gen Hosp Psychiatry 2007;29:182-91.

18. Givens JL, Katz IR, Bellamy S, et al. Stigma and the acceptability of depression treatments among african americans and whites. $J$ Gen Intern Med 2007;22:1292-7.

19. Nahin RL, Barnes PM, Strussman BJ. Expenditures on Complementary Health Approaches: United States. 2012. Atlanta GA: National Health Statistics Reports, 2016.

20. Kessler RC, Soukup J, Davis RB, et al. The use of complementary and alternative therapies to treat anxiety and depression in the United States. Am J Psychiatry 2001;158:289-94.

21. Cochrane Depression, Anxiety, and Neurosis Group. CCDAN Topic list: intervention - Psychological therapies. London: The Cochrane Collaboration, 2013. http://cmd.cochrane.org/sites/cmd.cochrane. org/files/public/uploads/CCDAN\%20topics\%20list_psychological\% 20therapies\%20for\%20website_0.pdf. (accessed 5 Jul 2016)

22. Higgins JPT, Green S, eds. Cochrane Handbook for Systematic Reviews of Interventions Version 5.1.0: The Cochrane Collaboration, 2011.

23. Shojania KG, Sampson M, Ansari MT, et al. How quickly do systematic reviews go out of date? A survival analysis. Ann Intern Med 2007;147:224-33.

24. Jorm A, Allen N, Morgan A, et al. A guide to what works for depression. Melbourne: Beyondblue, 2013. http://resources. beyondblue.org.au/prism/file?token=BL/0556. (accessed 22 Oct 2016)

25. Shea BJ, Hamel C, Wells GA, et al. AMSTAR is a reliable and valid measurement tool to assess the methodological quality of systematic reviews. J Clin Epidemiol 2009;62:1013-20.

26. Fu R, Gartlehner G, Grant M, et al. Conducting quantitative synthesis when comparing medical interventions: AHRQ and the Effective Health Care Program. J Clin Epidemiol 2011;64:1187-97.

27. Hedges LV. Distribution theory for Glass'sestimator of effect size and related estimators. Journal of Educational and Behavioral Statistics 1981;6:107-28.

28. Balshem $\mathrm{H}$, Helfand $\mathrm{M}$, Schünemann $\mathrm{HJ}$, et al. GRADE guidelines: 3 . Rating the quality of evidence. J Clin Epidemiol 2011;64:401-6.

29. Berkman ND, Lohr KN, Ansari MT, et al. Grading the strength of a body of evidence when assessing health care interventions: an EPC update. J Clin Epidemiol 2015;68:1312-24.

30. Yeung WF, Chung KF, Ng KY, Ky N, et al. A systematic review on the efficacy, safety and types of chinese herbal medicine for depression. J Psychiatr Res 2014;57:165-75.

31. Jun JH, Choi TY, Lee JA, et al. Herbal medicine (Gan Mai da Zao decoction) for depression: a systematic review and meta-analysis of randomized controlled trials. Maturitas 2014;79:370-80.
32. Appleton KM, Sallis HM, Perry R, et al. Omega-3 fatty acids for depression in adults. The Cochrane Database of Systematic Reviews 2015;11:CD004692.

33. Sorbero ME, Reynolds K, Colaiaco B, et al; Acupuncture for major depressive disorder. A systematic review. Santa Monica, CA: RAND Corporation, 2015

34. Linde K, Kriston L, Rücker G, et al. Efficacy and acceptability of pharmacological treatments for depressive disorders in primary care: systematic review and network meta-analysis. Ann Fam Med 2015;13:69-79.

35. Undurraga J, Baldessarini RJ, Randomized BRJ. Randomized, placebo-controlled trials of antidepressants for acute Major depression: thirty-year meta-analytic review. Neuropsychopharmacology 2012;37:851-64.

36. Josefsson T, Lindwall M, Archer T. Physical exercise intervention in depressive disorders: meta-analysis and systematic review. Scand $J$ Med Sci Sports 2014;24:259-72.

37. Liu X, Clark J, Siskind D, et al. A systematic review and metaanalysis of the effects of Qigong and Tai Chi for depressive symptoms. Complement Ther Med 2015;23:516-34.

38. Okumura Y, Ichikura K. Efficacy and acceptability of group cognitive behavioral therapy for depression: a systematic review and metaanalysis. J Affect Disord 2014;164:155-64.

39. Ekers D, Webster L, Van Straten A, et al. Behavioural activation for depression; an update of meta-analysis of effectiveness and sub group analysis. PLoS One 2014;9:e100100.

40. Abbass AA, Kisely SR, Town JM, et al. Short-term psychodynamic psychotherapies for common mental disorders. Cochrane Database Syst Rev 2014;7:CD004687.

41. Cuijpers P, Turner EH, Mohr DC, et al. Comparison of psychotherapies for adult depression to pill placebo control groups: a meta-analysis. Psychol Med 2014;44:685-95.

42. van Marwijk H, Allick G, Wegman F, et al. Cochrane Database of Systematic Reviews 2012;7 http://onlinelibrary.wiley.com/doi/.

43. Taylor D, Sparshatt A, Varma S, et al. Antidepressant efficacy of agomelatine: meta-analysis of published and unpublished studies. BMJ 2014;348:91888

44. Gartlehner G, Gaynes B, Amick H, et al. Nonpharmacological versus pharmacological treatments for adult patients with major depressive disorder. Rockville, MD: 2015.

45. Al-Karawi D, Jubair L. Bright light therapy for nonseasonal depression: meta-analysis of clinical trials. J Affect Disord 2016;198:64-71.

46. Apaydin EA, Maher AR, Shanman R, et al. A systematic review of St. John's wort for major depressive disorder. Syst Rev 2016;5:148.

47. Furukawa TA, Weitz ES, Tanaka S, et al. Initial severity of depression and efficacy of cognitive-behavioural therapy: individual-participant data meta-analysis of pill-placebo-controlled trials. Br J Psychiatry 2017:210:190-6.

48. Galizia I, Oldani L, Macritchie K, et al. S-adenosyl methionine (SAMe) for depression in adults. Cochrane Database Syst Rev 2016;10:CD011286.

49. Sorbero ME, Reynolds K, Colaiaco B, et al. Acupuncture for major depressive disorder. A systematic review: RAND National Defense Research Institute, 2015.

50. Gartlehner G, Gaynes BN, Amick HR, et al. Comparative benefits and harms of antidepressant, psychological, complementary, and exercise treatments for major depression: an evidence report for a clinical practice guideline from the American College of Physicians. Ann Intern Med 2016;164:331-41.

51. Turner EH, Matthews AM, Linardatos E, et al. Selective publication of antidepressant trials and its influence on apparent efficacy. $N$ Engl $J$ Med 2008;358:252-60.

52. Kirsch I, Deacon BJ, Huedo-Medina TB, et al. Initial severity and antidepressant benefits: a meta-analysis of data submitted to the Food and Drug Administration. PLoS Med 2008;5:e45.

53. Furukawa TA, Cipriani A, Atkinson LZ, et al. Placebo response rates in antidepressant trials: a systematic review of published and unpublished double-blind randomised controlled studies. Lancet Psychiatry 2016;3:1059-66.

54. Amick HR, Gartlehner G, Gaynes BN, et al. Comparative benefits and harms of second generation antidepressants and cognitive behavioral therapies in initial treatment of major depressive disorder: systematic review and meta-analysis. BMJ 2015;351:h6019.

55. Loh A, Leonhart R, Wills CE, et al. The impact of patient participation on adherence and clinical outcome in primary care of depression. Patient Educ Couns 2007;65:69-78.

56. Melartin TK, Rytsälä HJ, Leskelä US, et al. Continuity is the main challenge in treating major depressive disorder in psychiatric care. $J$ Clin Psychiatry 2005;66:220-7. 